\title{
Vortex-antivortex wavefunction of a degenerate quantum gas.
}

\author{
A.Yu.Okulov* \\ General Physics Institute of Russian Academy of Sciences Vavilova str. 38, 119991, Moscow, Russia
}

(Dated: March 1, 2009)

\begin{abstract}
A mechanism of a pinning of the quantized matter wave vortices by optical vortices in a specially arranged optical dipole traps is discussed. The vortex-antivortex optical arrays of rectangular symmetry are shown to transfer angular orbital momentum and form the "antiferromagnet"-like matter waves. The separable Hamiltonian for matter waves in pancake trapping geometry is proposed and 3D-wavefunction is factorized in a product of wavefunctions of the $1 \mathrm{D}$ harmonic oscillator and $2 \mathrm{D}$ vortex-antivortex quantum state. The $2 \mathrm{D}$ wavefunction's phase gradient field associated via Madelung transform with the field of classical velocities forms labyrinth-like structure. The macroscopic quantum state composed of periodically spaced counter-rotating BEC superfluid vortices has zero angular momentum and nonzero rotational energy.
\end{abstract}

PACS numbers: $42.50 . \mathrm{Tx}$ 42.65.Hw 42.65.Es 42.65.Sf

\section{INTRODUCTION.}

Ultracold atomic gases [1, 2] have attracted significant interest nowadays as a quantum simulators of condensed matter systems [3] and as an effective instrument for quantum information processing [4]. The basic physical mechanisms for control of atomic motion are magnetic trapping [5] and optical dipole trapping [6]. Different geometries of trapping fields were considered already, from the simplest one, based upon 1D sinusoidal standing wave, formed by two counter-propagating laser beams, to 3D artificial kagome potential landscape built by specially arranged tilted laser beams configuration [3]. Such an artificial potential lattices provide a rich opportunities for analog modeling of many-body quantum systems e.g. Mott insulator transition [7], quantum Hall effect [8 10], frustrated quantum antiferromagnets [3]. The antiferromanetic phase is considered as an essential counterpart of high temperature superconductivity (HTSC) [11]. Recently the persistent currents in toroidal "blue" detuned traps were reported [12]. The pinning of the co-directed superfluid vortices in different potential configurations was analyzed with variational wavefunctions in order to calculate vortex interaction energies and it was shown that the most favorable allocations of vortices are at maxima of the lattice potential [13]. On the other hand the elementary excitations of trapped ultracold gases, namely abelian and nonabelian anyons were proposed as a promising tool for error-tolerant quantum computing [14]. Noteworthy the recent the mutual control of the matter waves by light and vice versa demonstrated recently [16].

The interesting feature of an optical trapping by laser beams with wavefront dislocations is a possibility of guiding an atomic motion via non-potential optical fields i.e.

*Electronic address:

okulov@kapella.gpi.ru by virtue of the optical vortices. Initially the elementary optical vortices like Laguerre-Gaussian beams (LG) were considered as toroidal traps for red detuned cold atoms [17] or hollow "tubes" under blue detuning from resonance [18, 19]. For a red detuned trap of toroidal geometry [17] the optical torque had been predicted [20] which leads to angular acceleration of trapped atoms. In the absence of optical torque the macroscopic quantum state of BEC in toroidal trap had been studied by variational approach and a wavefunction in the form of LG vortex spiral was obtained 21]. Classical dynamics of an atom trapped by helical EM-fields guided by a nano-fiber has been shown recently to exhibit spiral motion outwards the beam axis as a result of optical torque 22]. The formation and acceleration of matter wave solitons in toroidal quasi-1D ring trap due to effect of an azimuthal oscillating electric field had been studied [23]. The vortex-antivortex pairs in two-transverse dimensions in non-rotating BEC traps of pancake geometry were obtained numerically [24]. The subject of the present article is an investigation of the structure of a BEC wavefunction in non - potential spatially periodic field composed of overlapping optical vortices 25. The paper is organized as follows. Section 2 describes the optical dipole trap composed of overlapping LG vortices. In section 3 the results for conservative (potential or gradient) and dissipative (radiation pressure) forces on two-level atom with electrical dipole transition are summarized. Section 4 connects the classical tensors of electromagnetic momentum and angular momentum with forces and torques on moving atom. In Section 5 the procedure of separation of variables for 3D Gross-Pitaevskii equation (GPE) is outlined along with numerical solution for $2 \mathrm{D}$ vortexantivortex wavefunction. The procedure of separation of variables is closely connected to existence of different spatial scales in GPE for pancake trap geometry, namely longitudinal $\ell_{z}$, transversal $\ell_{\perp}$ and healing length $\xi$. The healing length $\xi$ appears as effective nonlinear scale related to cubic term in GPE [2] as a condition of a balance between kinetic energy ("quantum pressure") and inter- 


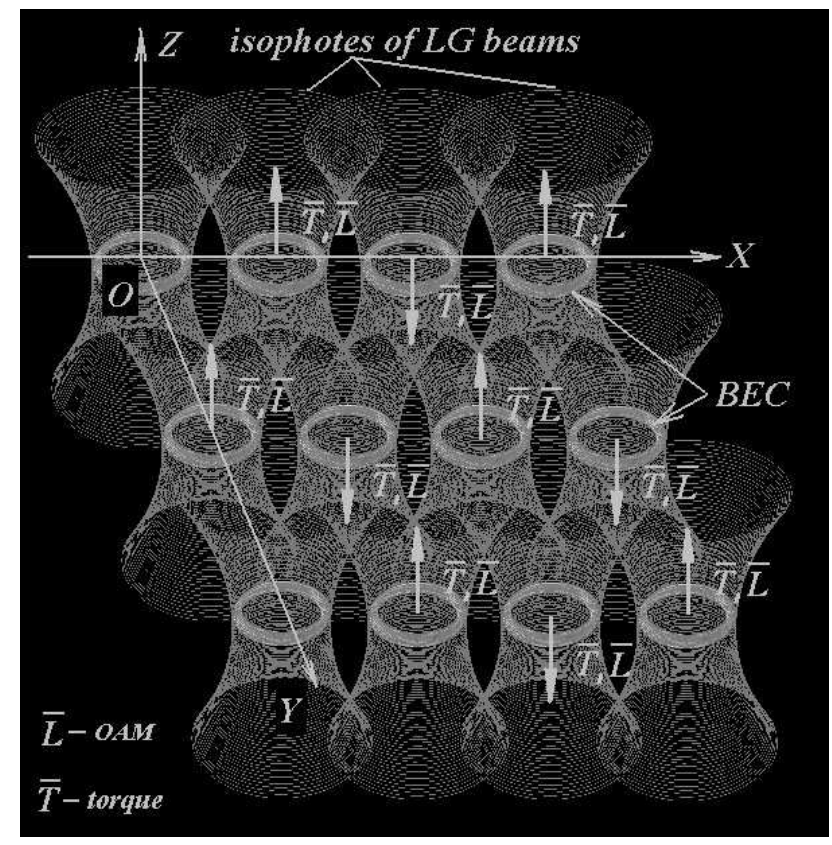

FIG. 1: The isointensity lines for the superposition of toroidal optical traps. Each elementary trap is $\mathbf{L G}$ beam propagating along $Z$ - axis. The hyperboloidal surfaces are the loci of the maxima of intensity. The rings at the LG beams bottlenecks are the isophotes which corresponds to a maxima of a light intensity. Their diameter is chosen close to experimentally observed in [29], namely $d \approx 30 \mu \mathrm{m}$, wavelength $\lambda \approx 0.8 \div 1.06 \mu \mathrm{m}$, $\mathrm{D} \approx 180 \mu \mathrm{m}$.

action energy (two-body interaction):

$$
\xi=\sqrt{1 / 8 \pi n a_{s}}
$$

where $n \approx|\Psi|^{2}$ is average density of a quantum gas, $a_{s}$ is the $s$-wave scattering length. The $\ell_{z, \perp}=\sqrt{\left(\hbar / m \omega_{z, \perp}\right)}$ are often referred to as a characteristic widths (longitudinal and transversal respectively) of the ground state of harmonic oscillator for parabolic traps. In pancake geometry considered below the following inequality is valid [26, 27]:

$$
\ell_{z}<\ell_{\perp}<\xi
$$

This allows to separate $z$ and $\vec{r}_{\perp}$ variables in GPE and factorize [23, 25, 28] or in other terms employ multiple scale expansion [26] to the $3 \mathrm{D}$ wavefunction $\Psi$ to decouple it in a product of longitudinal $\Psi_{z}$ and transversal $\Psi_{\perp}$ wavefunctions. Section 6 devoted to estimation of rotational energy and angular momentum of spatially periodic macroscopic quantum state and section 7 summarizes the obtained results.

\section{CONFIGURATION OF AN OPTICAL LABYRINTH TRAP FOR NEUTRAL ATOMS.}

The rectangular optical vortex lattices are spontaneously formed in diode-pumped microchip lasers with slightly focusing output coupler in a wide range of experimental parameters [29]. The optical patterns observed in this experiment are nonlinear eigenmodes of Fabry-Perot resonator with sufficiently large Fresnel number $N_{f r}=$ $\frac{k D^{2}}{L_{r}}$, ranging from 100 to 1000 : , where $k=2 \pi / \lambda$, $L_{r}$ is optical length of cavity, $D$ is diameter of optically pumped area inside host crystal which is approximately equal to diameter of generated optical array. It is convenient to approximate the laser eigenmode obtained numerically [30] and experimentally [29] as a superposition of co-propagating and overlapping LG's with the unit topological charges [25]:

$$
\begin{aligned}
& E(\vec{r}, z=0) \approx E_{0} \exp \left[-\frac{|\vec{r}|^{2}}{D^{2}}\right] \sum_{j x, j y}(-1)^{j x+j y}\left|\vec{r}-\vec{r}_{j x, j y}\right| \\
& \quad \times \exp \left[-\frac{\left|\vec{r}-\vec{r}_{j x, j y}\right|^{2}}{d^{2}}+i \ell_{E M} \operatorname{Arg}\left(\vec{r}-\vec{r}_{j x, j y}\right)\right],
\end{aligned}
$$

where $j x, j y$ are integer indices for positions of elementary LG vortices spanned with period $p$ in $(z=0, x, y)$ - plane, $d$ is diameter of the bottleneck of LG and $\ell_{E M}$ is topological charge of elementary LG optical vortex, $\vec{r}=(x, y)$ is a vector in transverse plane, $\vec{r}_{j x, j y}$ is a vector indicating positions of elementary vortices. The resulting interference pattern obtained via superposition (3) is ordered in "antiferromagnet"-like lattice with angular momenta alternating from one site to another(fig.1) [31]. Such 2D periodic optical vortex array forms a superposition of multiply connected toroidal optical traps (fig. 2). The motion of cold atoms is controlled by combined action of optical dipole force [6] and radiation pressure force [20, 22]. Alternative useful approximation for optical field is is a superposition of the several major Fourier components [34] using e.g. exact formula for free space propagation of periodical optical field from [35]:

$$
\begin{gathered}
E(\vec{r}, z) \approx E_{0} \exp \left[i k z-\frac{|\vec{r}|^{2}}{D^{2}}\right] \sum_{j x, j y} A_{j x, j y} \\
\exp \left[i 2 \pi\left\{\frac{x \cdot j x}{p}+\frac{y \cdot j y}{p}+\frac{z}{2 k}\left(\frac{j x^{2}}{p^{2}}+\frac{j y^{2}}{p^{2}}\right)\right\}\right] .
\end{gathered}
$$

It is worth to mention here that a similar geometry of the optical array produced with the programmable spatial light modulators [32] and microlens arrays [33] giving the possibility of fine tuning of phases $\phi_{j x, j y}$ and amplitudes $A m p\left(\vec{r}-\vec{r}_{j x, j y}\right)$ of a given beam in array:

$$
\begin{array}{r}
E(\vec{r}, z=0) \approx E_{0} \sum_{j x, j y} \operatorname{Amp}\left[\left(\vec{r}-\vec{r}_{j x, j y}\right) / d^{2}\right] \\
\exp \left[i \ell_{E M} \operatorname{Arg}\left(\vec{r}-\vec{r}_{j x, j y}\right)+i \phi_{j x, j y}\right],
\end{array}
$$




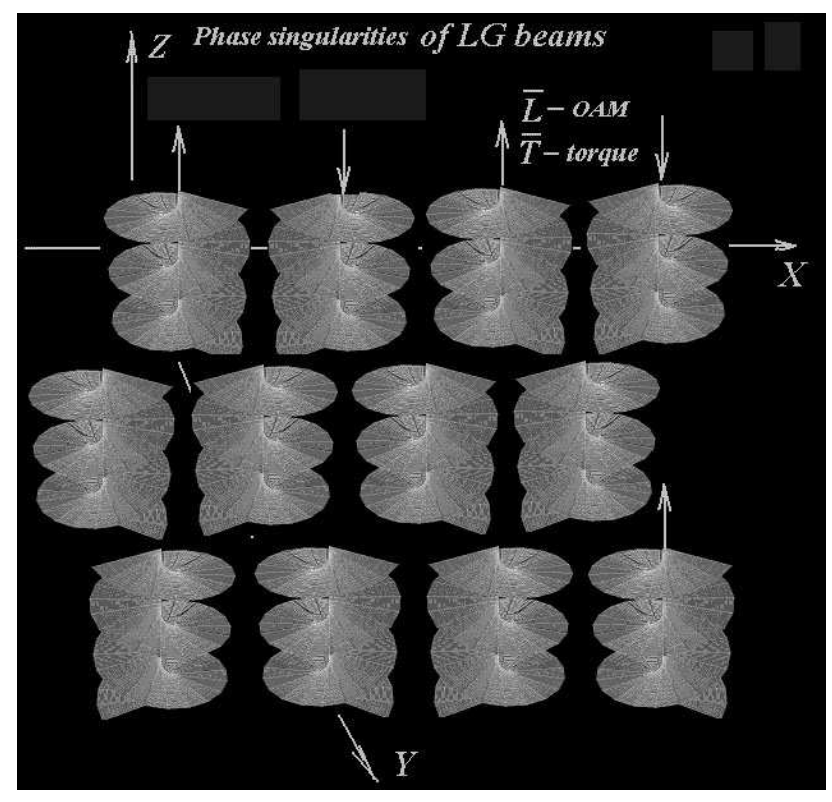

FIG. 2: The schematic representation of the periodically spaced optical vortices. Letters $\vec{L}$ and $\vec{T}$ denote the angular momentum carried by each phase singularity and torque, respectively. The directions of $\vec{T}, \vec{L}$ are shown to be parallel or anti-parallel to $Z$ - axis. The optical torques induce rotations of the cold atoms clockwice or counter - clockwice respectively. The helical surfaces are the snapshots of the phase of optical field at a given moment. The loci of helices are collocated with phase singularities. The optical phase is undetermined along the axes of helices denoted by arrows. One round trip around the axis of a given helix in the $x, y$ - plane means the $2 \pi$ change of the optical phase. The perfect match of helical wavefronts between adjacent vortices is seen clearly. The trapped dipole moves upstairs the helicoid, the radius of rotation is gradually increased due to torque. The passage from one helix to another is possible due to perfect match of adjacent helical surfaces.

\section{CONSERVATIVE AND DISSIPATIVE FORCES ON MOVING NEUTRAL ATOMS.}

The knowledge of a particular spatial distribution of $\vec{E}(z, \vec{r})$ permits the explicit calculation of the expectation values of $\hat{\vec{F}}$ i.e. classical force $\vec{F}$ on resonant atom with an electrical dipole transition. For conservative (or reactive) part $\vec{F}_{R}$ we have expression for gradient force $[6]$ :

$$
\langle\vec{F}\rangle_{R}=-\nabla\{\langle\hat{\vec{d}} \cdot \vec{E}(z, \vec{r})\rangle\}
$$

For dissipative component [20] the formula for radiation pressure follows:

$$
\begin{array}{r}
\langle\vec{F}\rangle=\langle\vec{F}\rangle_{R}+\langle\vec{F}\rangle_{D}=\frac{d\left\langle\vec{P}_{a t}\right\rangle}{d t}= \\
\frac{d}{d t}\left\{\left\langle\vec{P}_{a t}(t=0)+\frac{i}{\hbar} \int_{0}^{t}\left[\hat{H}\left(t^{\prime}\right), \vec{P}_{a t}\left(t^{\prime}\right)\right] d t^{\prime}\right\rangle\right\}= \\
i \hbar\left\{n\left(2 n_{e}-1\right)+n_{e}\right\}\left[f^{*} \nabla f I_{S}(t)-f \nabla f^{*} I_{S}^{*}(t)\right],
\end{array}
$$

where $\hat{\overrightarrow{P_{a t}}}$ is an atomic momentum, $\hat{H}\left(t^{\prime}\right)$ is a Hamiltonian of the atom in rotating wave approximation, $n_{e}=$ $\left\langle\Psi\left|\hat{n_{e}}\right| \Psi\right\rangle$ is an average number of atoms on upper level, $n=\left\langle\Psi\left|\hat{a}_{0}^{+} \hat{a_{0}}\right| \Psi\right\rangle$ is the average number of photons in a given electromagnetic mode ( $\mathrm{LG}_{01}$ mode in our case),

$$
\begin{array}{r}
\left.f \cong \vec{D}_{12} \cdot \vec{E}_{(} z, \vec{r}\right)=G(z, \vec{r}) \exp (i \Theta(z, \vec{r})) \\
I_{S}(t)=\int_{0}^{t} \exp \left[i \Delta t^{\prime} / 2\right] \frac{\sin \left(\Delta t^{\prime} / 2\right)}{\Delta / 2}
\end{array}
$$

where $\vec{D}_{12}$ is electric dipole matrix element of the twolevel transition, $\Delta=\omega_{0}-\omega+\delta$ is a detuning and $\delta$ is given by:

$$
\delta=\frac{1}{2 M}\left[\frac{\langle\mathbf{P}\rangle \cdot \nabla f+\nabla f \cdot\langle\mathbf{P}\rangle}{f}\right]_{0} .
$$

Fortunately the back action of the radiation scattered by freely moving atom upon incident field is negligibly small for currently achieved atom densities in optical dipole traps and a field $\vec{E}(\vec{r}, z)$ could be substituted from classical solution of Maxwell equations. As a result an azimuthal component of semiclassical dissipative force $\left\langle F_{\phi}\right\rangle_{D}$ on atom in LG helical optical beam is as follows [20]:

$$
\left\langle F_{\phi}\right\rangle_{D}=\frac{2 \hbar \Gamma \Omega^{2}{ }_{k p l}(\vec{r}, z)}{\Delta^{2}+2 \Omega^{2}{ }_{k p l}(\vec{r}, z)+\Gamma^{2}} \frac{\ell_{e m}}{r} \vec{\phi}
$$

where $\Omega^{2}{ }_{k p l}(\vec{r}, z)=\vec{D}_{12} \cdot \vec{E}(z, \vec{r}) / \hbar, \Gamma$ is atomic linewidth. This component is responsible for the angular acceleration of the atomic dipole around $Z$ - axis. Two other components of dissipative force were also obtained in explicit form. The longitudinal component of the radiation pressure force is:

$$
\left\langle F_{z}\right\rangle_{D}=\frac{\hbar k \Gamma I(\vec{r}, z)}{1+I(\vec{r}, z)+\Delta^{2} / \Gamma^{2}},
$$

and radial force $\left\langle F_{r}\right\rangle_{D}$ is :

$$
\left\langle F_{r}\right\rangle_{D}=\frac{\hbar k \Gamma \nabla I(\vec{r}, z) \vec{r}}{2 \epsilon_{0} c}\left[\frac{1}{1+I(\vec{r}, z)+\Delta^{2} / \Gamma^{2}}\right]
$$


As a result complete $3 D$ classical motion of the atom with resonant dipole transition having mass $m$ in isolated optical vortex is governed by following equations [22]:

$m \ddot{z}=F_{z} \quad ; \quad m \ddot{r}=F_{r}+m r \dot{\phi}^{2} \quad ; \quad m r \ddot{\phi}=-2 m \dot{r} \dot{\phi}+F_{z}$.

On the other hand in vector notations we have the following equation for classical motion of a particle with a mass $m$ :

$$
\dot{\vec{L}}=\frac{d}{d t}\left\{m \vec{r}^{2} \vec{\omega}\right\}=\vec{T}(\vec{r})=[\vec{r} \times \vec{F}]
$$

The straitforward generalization of this equation for rectangular array of equidistantly spanned superimposed vortices reads:

$$
\begin{array}{r}
m \ddot{\vec{r}}=\sum_{j x, j y} \vec{F}\left(\vec{r}-\vec{r}_{j x, j y}, z\right) ; \\
m \ddot{\vec{r}}=\sum_{j x \pm 1, j y \pm 1} \vec{F}\left(\vec{r}-\vec{r}_{j x, j y}, z\right),
\end{array}
$$

where second equation in (15) takes into account only nearest neighboring overlapping optical vortices. The azymuthal components of the Pointing vector accelerates the condensate around vortex axis (fig. 2 Z-axis) in the following way. The classical trajectories initially located near vortex core with velocities close to zero are almost circular. The radius of rotation is gradually increased until atom would reach the separatrix, analogously to [22]. Then atom passes to another basin of atraction located around ajaicent vortex core[25].The numerical solution of (15) shows how classical dipole trapped in optical labyrinth field moves along Mobius-like trajectories around zeros of intensity, roaming from one phase singularity to another.

\section{DENSITIES OF THE LINEAR AND ANGULAR ELECTROMAGNETIC MOMENTA}

Using effective cross-section $\sigma_{o p t}$ of an atom which scatters the optical field $\vec{E}(\vec{r}, t)$, the equations (10, 12) could be reformulated in a terms of electromagnetic energy flux (Pointing vector) $\vec{S}=\epsilon_{0} c^{2}[\vec{E} \vec{B}]$, momentum density $\vec{P}(\vec{r}, t)$, momentum flux density $T^{j l}$, angular momentum density $\vec{M}(\vec{r}, t)$, angular momentum flux density $M^{j l}$ [36]. Classically the force $d \vec{F}$ experienced by infinitesimal element of surface of a physical body in electromagnetic field could be evaluated by multiplying of the electromagnetic pressure $p$ by an infinitesimally small surface element $d s$ having local normal $\vec{n}(\vec{R})$ :

$$
d \vec{F} \approx p \vec{n}(\vec{R}) d s,
$$

The physical meaning of expression (16) is momentum flux through infinitesimal surface element $d s$ of area per a unit time. Taking into account only normal component of optical flux i.e. component parallel to normal $\vec{n}$ the force $d \vec{F}$ on this particular surface element in tensor notations is as follows: $d F_{j}=T^{j l} d s_{l}$,

where $T^{j l}=\frac{\delta_{j l} l}{2}\left[\epsilon_{0}|\vec{E}|^{2}+\mu_{0}^{-1}|\vec{B}|^{2}\right]-\epsilon_{0} E_{j} E_{l}-$ $\mu_{0}{ }^{-1} B_{j} B_{l}$ is momentum flux density [36], $\delta_{j l}$ is Kronecker's delta. The magnitudes of $T^{j l}$ components define the magnitudes and directions of optical forces on atoms in the vicinity of phase singularity (fig. 2). The components of the energy flux density (Pointing vector) $\vec{S}=\epsilon_{0} c^{2}[\vec{E} \times \vec{B}]$ are proportional to linear momentum density $\vec{P}=\vec{S} / c^{2}=\epsilon_{0}[\vec{E} \times \vec{B}]$ components:

$$
\begin{gathered}
P_{z}=2 \epsilon_{0} c|\vec{E}(\vec{r}, z)|^{2} \quad ; \quad P_{r}=\epsilon_{0} \frac{\omega k r z}{z^{2}+z_{R}^{2}}|\vec{E}(\vec{r}, z)|^{2} \\
P_{\phi}=\epsilon_{0}\left[\frac{\omega \ell_{e m}}{r}|\vec{E}(\vec{r}, z)|^{2}-\frac{\omega \sigma}{2} \frac{\partial|\vec{E}(\vec{r}, z)|^{2}}{\partial r}\right],
\end{gathered}
$$

where $\sigma$ is a light polarization equal to $0, \pm 1$ for plane and circular polarizations respectively [37]. The major, i.e. longitudinal component $P_{z}$ (17) is responsible for optical pressure force. The radial component $P_{r}$ (17) pushes atomic dipole outwards the beam axis $(Z)$ and the last, azimuthal component $P_{\phi}$ of (17) accelerates atom around $Z$ - axis. Angular momentum density $\vec{M}$ is defined as a vector product, analogously to definition of mechanical torque $\vec{T}=[\vec{r} \times \vec{F}]$ :

$$
\begin{array}{r}
\vec{M}=[\vec{r} \times \vec{P}(\vec{r}, z)]=\frac{[\vec{r} \times \vec{S}(\vec{r}, z)]}{c^{2}}=\epsilon_{0} \vec{r} \times \\
{[\vec{E}(\vec{r}, z) \times \vec{B}(\vec{r}, z)]=-\frac{\ell_{e m} z}{\omega r}|\vec{E}(\vec{r}, z)|^{2} \vec{r}-} \\
\frac{r}{c}\left[\frac{z^{2}}{\left(z^{2}-z_{R^{2}}\right)}-1\right]|\vec{E}(\vec{r}, z)|^{2} \vec{\phi}+\frac{\ell_{e m}|\vec{E}(\vec{r}, z)|^{2}}{\omega z} \vec{z},
\end{array}
$$

where $z_{R}=k D^{2}$ is Rayleigh range. The field of transversal (in the plane $x, y$ ) momentum density $\vec{P}(x, y)$ is proportional to the phase gradient (fig, 3):

$$
\vec{P}(x, y) \approx \nabla\{\operatorname{Arg}[\vec{E}(x, y, z=0)]\}
$$

The interference between adjacent LG - beams generates additional optical vortex lattice with opposite angular momenta [25, 31]. Under conditions of the far detuning from resonance it is possible to construct the optical dipole potential. This potential is separable in the geometry considered in [25] (Fig. 1):

$$
\begin{array}{r}
V_{e x t}\left(r_{\perp}, z\right)=V_{z}+V_{\perp}=\frac{m \omega_{z}^{2} z^{2}}{2}- \\
\operatorname{Re}[\alpha(\omega)]\left|\vec{E}\left(\vec{r}_{\perp}\right)\right|^{2}+\frac{m \omega_{\perp}{ }^{2}\left|\left(\vec{r}_{\perp}\right)\right|^{2}}{2},
\end{array}
$$


where $\omega_{z}, \omega_{\perp}$ are the transversal and longitudinal frequencies of optical trap respectively[1]. $\alpha(\omega)$ is the polarizability of atom $[6]$ :

$$
\alpha(\omega)=6 \pi \epsilon_{0} c^{3} \frac{\Gamma / \omega_{0}^{2}}{\left(\omega_{0}^{2}-\omega^{2}-i\left(\omega^{3} / \omega_{0}^{2}\right) \Gamma\right)},
$$

which is real for large detuning from resonance $\omega-\omega_{0}$.

\section{GROSS-PITAEVSKII EQUATION WITH SEPARABLE HAMILTONIAN.}

We solve the Gross-Pitaevskii equation (GPE) for macroscopic BEC wavefunction [1, 2]:

$$
i \hbar \frac{\partial \Psi(\vec{r}, t)}{\partial t}=\hat{H} \Psi(\vec{r}, t)
$$

with following separable Hamiltonian [26 28] :

$$
\begin{array}{r}
\hat{H}=\hat{H}_{\perp}+\hat{H}_{\|}=-\frac{\hbar^{2}}{2 m} \Delta_{\perp}-\frac{\hbar^{2}}{2 m} \frac{\partial^{2}}{\partial z^{2}}+\frac{m \omega_{z}^{2} z^{2}}{2}- \\
\operatorname{Re}[\alpha(\omega)]\left|E\left(\vec{r}_{\perp}\right)\right|^{2}+\frac{m \omega_{\perp}^{2}\left|\left(\vec{r}_{\perp}\right)\right|^{2}}{2}+ \\
\frac{4 \pi \hbar^{2} a_{s}(\vec{B})}{m}|\Psi(\vec{r}, t)|^{2},
\end{array}
$$

where $a_{s}$ is $s$-wave scattering length. For the asymmetrical optical trap when $\omega_{\perp}<\omega_{z}$ and when "healing length" $\xi=\left(8 \pi n a_{s}\right)^{-1 / 2}[2]$ is larger than longitudinal harmonic oscillator length $\sqrt{\hbar / m \omega_{z}}$ [26, 27]. Hence it is reasonable to seek the solution for the eq.(22) with Hamiltonian (23) by method of separation of variables [28]. The substitution of the factorized wavefunction $\Psi\left(z, r_{\perp}, t\right)=\Psi_{\perp}\left(r_{\perp}, t\right) \Psi_{\|}(z, t)$ in (22) gives:

$$
\begin{array}{r}
i \hbar\left[\Psi_{\|} \frac{\partial \Psi_{\perp}}{\partial t}+\Psi_{\perp} \frac{\partial \Psi_{\|}}{\partial t}\right]=-\Psi_{\|} \frac{\hbar^{2}}{2 m} \Delta_{\perp} \Psi_{\perp}- \\
\operatorname{Re}[\alpha(\omega)]\left|E\left(\vec{r}_{\perp}\right)\right|^{2} \Psi_{\|} \Psi_{\perp}-\frac{\hbar^{2}}{2 m} \frac{\partial^{2} \Psi_{\|}}{\partial z^{2}} \Psi_{\perp}+\frac{m \omega_{z}^{2} z^{2}}{2} \Psi_{\|} \Psi_{\perp} \\
+\frac{m \omega_{\perp}{ }^{2}\left|\left(\vec{r}_{\perp}\right)\right|^{2}}{2} \Psi_{\|} \Psi_{\perp}+\frac{4 \pi \hbar^{2} a_{s}(\vec{B})}{m} \Psi_{\perp}\left|\Psi_{\perp}\right|^{2} \Psi_{\|}\left|\Psi_{\|}\right|^{2} .(24)
\end{array}
$$

As a consequence of the different spatial scales $\ell_{z}<$ $\ell_{\perp}<\xi$ the starting GPE (22) is exactly decoupled in a way analogous to [26, 28]:

$$
i \hbar \Psi_{\perp} \frac{\partial \Psi_{\|}}{\partial t}=-\Psi_{\perp} \frac{\hbar^{2}}{2 m} \frac{\partial^{2} \Psi_{\|}}{\partial z^{2}}+\Psi_{\perp} \frac{m \omega_{z}^{2} z^{2}}{2} \Psi_{\|}
$$

and

$$
\begin{gathered}
i \hbar \Psi_{\|} \frac{\partial \Psi_{\perp}}{\partial t}=-\Psi_{\|} \frac{\hbar^{2}}{2 m} \Delta_{\perp} \Psi_{\perp}-\operatorname{Re}[\alpha(\omega)]\left|E\left(\vec{r}_{\perp}\right)\right|^{2} \Psi_{\|} \Psi_{\perp}+ \\
\frac{m \omega_{\perp}^{2}\left|\left(\vec{r}_{\perp}\right)\right|^{2}}{2} \Psi_{\|} \Psi_{\perp}+\frac{4 \pi \hbar^{2} a_{s}(\vec{B})}{m} \Psi_{\perp}\left|\Psi_{\perp}\right|^{2} \Psi_{\|}\left|\Psi_{\|}\right|^{2} .
\end{gathered}
$$

The solution of (25) for ground state inside longitudinal parabolic trap (harmonic oscillator) becomes evident:

$$
\Psi_{\|}=\left(\frac{m \omega_{z}}{\pi \hbar}\right)^{1 / 4} \exp \left[-m \omega_{z} z^{2} /(2 \hbar)-i \omega_{z} t\right]
$$

Next, by virtue of multiplying (26) by complex conjugate $\Psi_{\|}$, integrating it by $z$ from $-\infty$ to $\infty$, i.e. using normalization conditions:

$$
\int_{-\infty}^{\infty}\left|\Psi_{\|}(z, t)\right|^{4} d z=1 / 2 \text { and } \int_{-\infty}^{\infty}\left|\Psi_{\|}(z, t)\right|^{2} d z=1
$$

one obtains:

$$
\begin{array}{r}
i \hbar \frac{\partial \Psi_{\perp}}{\partial t}=-\frac{\hbar^{2}}{2 m} \Delta_{\perp} \Psi_{\perp}+\operatorname{Re}[\alpha(\omega)]\left|E\left(\vec{r}_{\perp}\right)\right|^{2} \Psi_{\perp}+ \\
\frac{4 \pi \hbar^{2} a_{s}(\vec{B})}{m} \Psi_{\perp}\left|\Psi_{\perp}\right|^{2}
\end{array}
$$

Next the transversal component of wavefunction is obtained numerically via split-step FFT algorithm on $512 \times 512$ points square computational mesh [25]. In order to emulate the optical torque not included yet in our computational model we prepared a special initial conditions for the transversal wavefunction $\Psi_{\perp}\left(t=0, \vec{r}_{\perp}\right)$ in the form of the rectangular array in the form of (4) and found the convergence with reasonable accuracy $\left(\approx 10^{-3}\right)$ after $200 \div 500$ iterates. It is worth to mention specially, that the rectangular symmetry of numerical solution of $2 \mathrm{D}$ GPE (29) is imposed by combined action of initial trial wavefunction $\Psi^{0}(\vec{r}, t)$ having rectangular symmetry similar to trapping potential and weakness of cubic term in (29) compared to trapping term. The initial guess for iterative explicit split-step FFT method was choosen in the form $2 \mathrm{D}$ vortex lattices (4). After $n_{i}=20 \div 150$ iterates the $\Psi^{n_{i}}(\vec{r}, t)$ remained well correlated with trapping field $E(\vec{r})$. Next within following $n_{i}=200 \div 500$ iterates the amplitude of wavefunction decreased down to $10 \div 100$ times smaller than initial guess amplitude, due to the intrinsic dissipation of the numerical method, which uses 4 the spatial filtering of the Fourrier components of high spatial frequencies [40, 41].

It is well known that spatially periodic optical trapping leads to Bloch waves and gaps in BEC energy spectrum. The gaps and cubic nonlinearity affect each other. In order to simplify numerical solution of (29) the parameters of equation were adjusted in such a way, that the last two terms in (29) would almost exactly cancel each other:

$$
\operatorname{Re}[\alpha(\omega)]\left|E\left(\vec{r}_{\perp}\right)\right|^{2} \Psi_{\perp}+\frac{2 \pi \hbar^{2} a_{s}(|\vec{B}|)}{m} \Psi_{\perp}\left|\Psi_{\perp}\right|^{2} \approx 0
$$

This condition could be fulfilled by virtue of the tuning scattering length $a_{s}$ via Feshbach resonance [2]: 


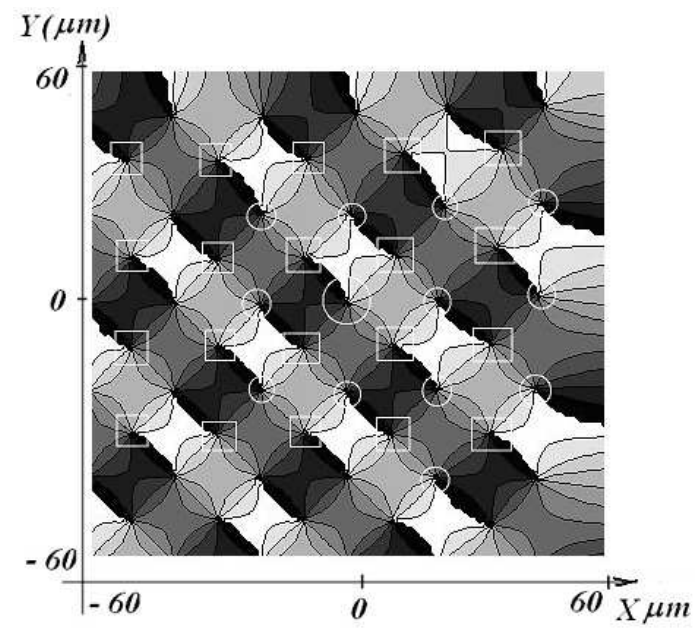

FIG. 3: The 2D plot of the phase of electric field $\operatorname{Arg}[E(\vec{r}, z=0)]$, eq. (3) in the $x, y$ - plane. The locations of the elementary optical vortices with positive topological charges $\ell=+1$ are labeled by white circles. The surrounding vortices having the opposite charges $\ell=-1$ are labeled by white squares. Such flip-flop distribution of local angular momentum arises due to interference of the overlapping LG beams in eq. (1).

$$
a_{s}(|\tilde{\mathbf{B}}|)=a_{s}^{b g}\left(1+\frac{\Delta_{B}}{B-B_{R}}\right)
$$

where $\Delta_{B}$ is a width of Feshbach resonance, $B_{R}$ is the value of the resonant magnetic field, $a_{s}{ }^{b g}$ is background value of s-wave scattering length $a_{s}$. The 3D solution $\Psi(\vec{r}, t)$ including numerical evaluation of (29) corresponds to "pancake"-like BEC cloud aligned in the vicinity of the $Z=0$ - plane (fig. 1). The superfluid vortices are collocated with the phase singularities of the optical field. In contrast to the rotating "bucket" trap [38, 39] and rotating "basket" trap [34], where superfluid vortex lattices rotate as a rigid body, our solution (fig. 4) is static. The superfluid vortices in our static "basket" trap proved to be pinned at the nodes (i.e. zeros of amplitude or phase singularities) of the optical interference pattern. The argument of $\Psi_{\perp}$ versus transverse coordinates shows clearly the loci of rectangularily spaced vortices with alternating circulations (fig. 5). The elementary superfluid vortices are labelled by white circles and squares. The topological charges $\ell$ of the vortices labelled by circles are $\ell=+1$, whereas the closely neighbouring squares have the opposite charges $\ell=-1$.

\section{ROTATIONAL ENERGY OF THE}

\section{VORTEX-ANTIVORTEX QUANTUM STATE.}

Following to Feynman [42] consider first the rotational energy of an isolated vortex. In contrast to the vortex in a classical liquid which rotates as a rigid body and

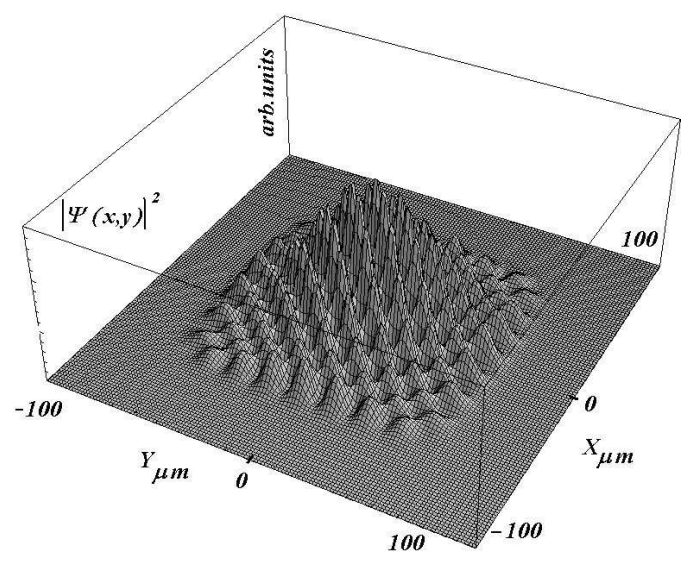

FIG. 4: The 2D plot of the square modulus of the macroscopic wavefunction $\Psi(\vec{r}, z=0)$ in the $x, y$ - plane.

have the constant angular velocity $\omega(r)=v(r) / r$, where $v(r)$ - is speed of flow line at the distance $r$ from vortex core, the quantum liquid rotates in such a way that the phase $\theta$ of wavefunction $\Psi$ remains single-valued. Because the argument of the wave function $\theta$ is connected with velocity $\vec{v}(\vec{r})$ of superfluid via Madelung transform $\nabla \theta(\vec{r}, t)=m \vec{v} / \hbar$, the contour integral $\oint \nabla \theta(\vec{r}, t) d \vec{l}=$ $2 \pi r m v / \hbar$ around the vortex core must be a multiple of $2 \pi$. As a consequence the quantization of angular momentum follows $m v r=\hbar$ because of a single-valued phase $\theta$ of the wavefunction $\Psi_{\perp}$. The next step is in evaluation of rotational kinetic energy of the vortex using the classical definition: $\int_{a}^{b} \omega^{2}(r) d J(r) / 2$. The moment of inertia of an infinitesimally thin $(d r)$ ring $d J(r)=r^{2} \rho 2 \pi r d r \chi$ rotating with angular velocity $\omega(r)=v / r=\hbar /\left(m r^{2}\right)$ is integrated from the inner radius $a$ of the vortex core to the external one $b$ :

$$
\begin{array}{r}
E_{\text {rot }}=\int_{a}^{b} \omega^{2}(r) d J(r) / 2= \\
\chi \rho \int_{a}^{b} \hbar^{2} \frac{\pi}{m r} d r= \\
\chi \hbar^{2} \rho \frac{\pi}{m} \ln (b / a),
\end{array}
$$

where $\chi$ is the length of vortex line in $z$-direction. Angular momentum of the vortex line may be determined by an analogous classical procedure:

$$
L_{v o r t}=\int_{a}^{b} \omega(r) d J(r)=\chi \pi \hbar \rho \int_{a}^{b} \frac{r d r}{m}=\chi \pi \hbar \rho \frac{b^{2}-a^{2}}{2 m}
$$

Vectorial nature of angular momentum $\vec{L}$ means that for a rectangular array of equispaced vortices with opposite circulations the local $\vec{L}_{i, j}$ are counter-directed (fig.). Thus the total angular momentum of the array tends to be equal to zero: 


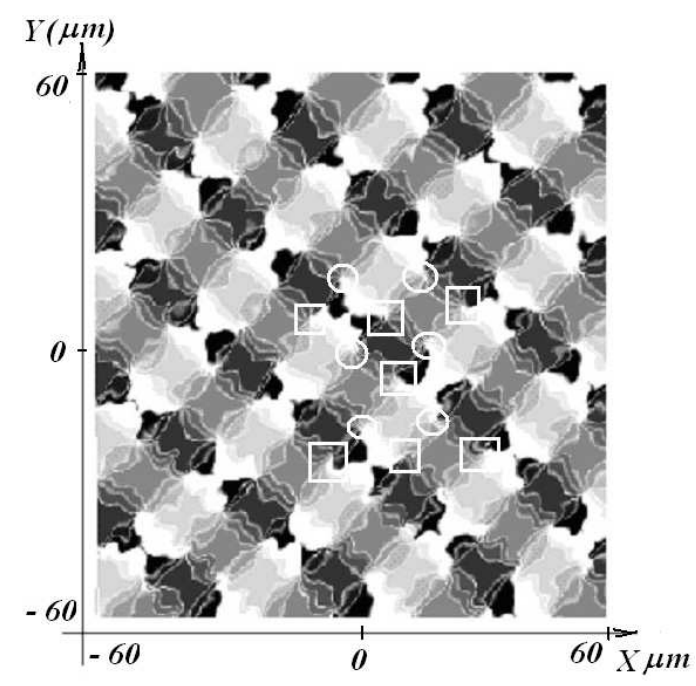

FIG. 5: The 2D plot of phase $\theta$ of macroscopic wavefunction $\Psi(\vec{r}, z=0)$ in the $x, y$ - plane. The locations of the condensate vortices with positive topological charges $\ell=+1$ are labeled by white circles. The vortices having the opposite charges $\ell=-1$ are labeled by white squares. $Z$ - axis is normal directed to reader.

$$
\vec{L}=\sum_{j x, j y} \vec{L}_{j x, j y} \approx 0 .
$$

On the other hand, the energies of the vortices are positive scalars hence their energy in a rotational ground state are additive values for noninteracting vortices:

$E_{\text {ground }}=\sum_{j x, j y} E_{j x, j y}=2 \times N_{\text {vortices }}^{2} \times \chi \hbar^{2} \rho \frac{\pi}{m} \ln (b / a)$
The quantum mechanical evaluation of the energy and angular momentum of vortex-antivortex quantum state is performed as follows [13]. By definition of quantum expectation values we have for the kinetic energy of the condensate [43]:

$$
\begin{array}{r}
E_{\text {ground }}=<\Psi^{*}|\hat{H}| \Psi>= \\
\chi \iint \Psi^{*}\left\{-\frac{\hbar^{2} \Delta_{\perp}}{2 m}+V\left(\vec{r}_{\perp}\right)+\frac{4 \pi \hbar^{2} a_{s}}{m}|\Psi|^{2}\right\} \Psi d^{2} \vec{r}_{\perp} \\
\approx N^{2}{ }_{\text {vortices }} \times \chi \hbar^{2} \rho \frac{\pi}{m} \ln (b / a),
\end{array}
$$

and for the angular momentum $L_{z}$ :

$$
L_{z}=\left\langle\Psi^{*}|\hat{L}| \Psi>=<\Psi^{*}\left|-i \hbar \frac{\partial}{\partial \phi}\right| \Psi>\approx 0\right. \text {. }
$$

\section{CONCLUSION.}

The outlined optical labyrinth trap setup is capable to support an asymmetrical cloud of ultracold atoms "red" detuned from resonance. The macroscopic quantum state obtained under facrorization conditions: the longitudinal component is $z$ - dependent gaussian with characteristic width of harmonic oscillator's ground state, while transversal part is periodic rectangular vortex lattice, pinned by the vortices of trapping optical lattice. The optical torque will be shown to cause the atoms roaming across optical lattice.
[1] L.Pitaevskii and S.Stringari, "Bose-Einstein Condensation" ( Clarendon Press, Oxford, 2003).

[2] F. Dalfovo, S.Giorgini, S.Stringari, L.P.Pitaevskii, Rev.Mod.Phys.71,463(1999).

[3] B.Damski,H.Fehrmann,H.H.Everts,M.Baranov,L.Santos, and M.Lewenstein, Phys.Rev.A72, 053612(2005).

[4] M.Fleischhauer,A.Imamoglu and J.P.Marangos, Rev.Mod.Phys.77, 633(2005). M.D.Lukin, Rev.Mod.Phys.75,457(2003).

[5] W.D.Phillips, Rev.Mod.Phys.70, 721-741(1998).

[6] R.Grimm,M.Weidemuller and Yu.B.Ovchinnikov, Adv.At.Mol. Opt.Phys. 42, 95(2000). V.S.Letokhov,JETP Lett,v.7,n.9,p. 272(1968).

[7] M. Greiner, O. Mandel, T. Esslinger, T.W. Hnsch, and I. Bloch ,Nature (London), 415, 39-44(2002).

[8] N. R. Cooper and N. K. Wilkin, Phys.Rev.B60, R16279 (1999).

[9] V. Schweikhard,I. Coddington, P. Engels, V. P. Mo- gendorff, and E. A. Cornell, Phys.Rev.Lett. 92, 040404(2004).

[10] T.L.Ho, Phys.Rev.Lett. 87, 060403(2001).

[11] E.G. Maksimov,O.V. Dolgov,Phys.Usp.50, n.9, p.933937(2007).

[12] K. Helmerson, M.F.Andersen, C.Ryu, P.Clade, P.Natarajan, A. Vaziri, and W.D.Philips, Nuclear Physics A 790, 705(2007).

[13] J.W.Reijnders and R.A.Duine, Phys.Rev.A71, 063607 (2005).

[14] M.H.Freedman,A.Kitaev,M.Larsen,Z.Wang, Bull.Am.Mat.Soc. 40, 31(2002).

[15] C.Nayak,S.H. Simon,A.Stern,M.H.Freedman,S.D.Sarma, LANL e-print 0707.1889v2[cond-mat.str-e1], http://arxiv.org, (2008).

[16] N.S.Ginsberg,S.R.Garner,L.V.Hau, Nature 445, 623626(2007).

[17] E. M. Wright, J. Arlt and K. Dholakia, Phys.Rev. A, 63, 
013608 (2001).

[18] V.I.Balykin and V.S.Letokhov,Sov.Phys.Usp.,33,79(1990). JETP,94,140(1988).

[19] T.Kuga,Y.Tori,N.Shiokawa,T.Hirano,Y.Shimizu, and H.Sasada, Phys.Rev.Lett. 78, 4713(1997).

[20] M. Babiker, W. L. Power, and L. Allen, Phys. Rev. Lett. 73, 1239 (1994).

[21] E.R.I.Abraham, J.Tempere and J.T.Devreese, Phys.Rev, 64A,023603 (2002).

[22] Fam Le Kien,V.I.Balykin and K.Hakuta,Phys. Rev.,A74,033412 (2006).

[23] Yu.V.Bludov and V.V.Konotop,Phys.Rev.A75,053614 (2007).

[24] L.C.Crasovan, V.Vekslerchik, V.M.Perez-Garcia, J.P.Torres, D.Mihalache, L.Torner, Phys.Rev. A 68, 063609 (2003).

[25] A.Yu.Okulov,J.Mod.Opt.,55,n2,241(2008).

[26] V.M.Perez-Garcia, H.Michinel and H.Herrero, Phys.Rev. A 57, 3837 (1998).

[27] R.Carretero-Gonzalez,D.J.Frantzeskakis, and P.G.Kevrekidis, Nonlinearity, 57, (2008).

[28] R.Fidele,P.K.Shukla,

S.De.Nicola, M.A.Manko,V.I.Manko,F.S.Cataliotti, JETP Lett, 80, 609 (2004).

[29] Y.F.Chen, Y.P.Lan, Phys.Rev, A64, 063807 (2001), Y. F. Chen,Y. P. Lan, Phys.Rev, A65, 013802 (2001).

[30] K.Staliunas, C.O.Weiss, JOSA B 12, 1142 (1995).
[31] A.Yu.Okulov, QCMC-2004, AIP Conference Proceedings, 734, 366(2004), A.Yu.Okulov, Bulletin Lebedev Physical Institute, n9, p.3, Sept.(2003).

[32] S.Bergamini, B.Darquie, M.Jones, L.Jacubowiez, A.Browaeys and P.Grangier. JOSA B, 21,1889,(2004).

[33] R.Dumke, M.Volk, T. Muther, F.B.J.Buchkremer, G. Birkl and W.Ertmer, Phys. Rev. Lett. 89, 097903 (2002).

[34] S.Tung, V.Schweikhard, and E.A.Cornell, Phys.Rev.Lett., 97, 240402 (2006).

[35] A.Yu.Okulov, JOSA B7, p.1045, (1990).

[36] S.M.Barnett, J. Opt. B,Quantum Semiclass. Opt., 4,S7S16 (2002).

[37] L.Allen, M.W.Beijersbergen, R.J.C.Spreeuw and J.P.Woerdman Phys.Rev. A, 45 p.8185-8189 (1992).

[38] J.R.Abo-Shaerr, C.Raman, J.M.Vogels and W.Ketterle, Phys. Rev. Lett.,88, 070409 (2002).

[39] I.Danaila, Phys.Rev.A72,013605 (2005).

[40] A.Yu.Okulov,Opt.Comm,99,p.350(1993). J.Mod.Opt.,38,N.10,p.1887(1991).

[41] A.Yu.Okulov,Optics and Spectroscopy., 77, n6, p.985 (1994).

[42] R.P.Feynman,"Statistical mechanics", (Ch.11, Reading, Massachusetts,1972).

[43] L.D.Landau and E.M.Lifshitz 1982 "Quantum Mechanics" (Pergamon Press, Tarrytown, New York, 1977), Vol. 3 . 